\title{
NOTES ON THE STARLIKE LOG-HARMONIC MAPPINGS OF ORDER ALPHA
}

\author{
R. KARGAR AND H. MAHZOON
}

\begin{abstract}
Let $h$ and $g$ be two analytic functions in the unit disc $\Delta$ that $g(0)=1$. Also let $\beta$ be a complex number with $\operatorname{Re}\{\beta\}>-1 / 2$. A function $f$ is said to be log-harmonic mapping if it has the following representation

$$
f(z)=z|z|^{2 \beta} h(z) \overline{g(z)} \quad(z \in \Delta) .
$$
\end{abstract}

A $\log$-harmonic mapping $f$ is said to be starlike log-harmonic mapping of order $\alpha$, where $0 \leq \alpha<1$, if

$$
\operatorname{Re}\left\{\frac{z f_{z}-\bar{z} f_{\bar{z}}}{f}\right\}>\alpha \quad(z \in \Delta) .
$$

In this paper, by use of the subordination principle, we study some geometric properties of the starlike log-harmonic mappings of order $\alpha$. Also, we estimate the Jacobian of log-harmonic mappings.

\section{INTRODUCTION}

Let $\mathcal{H}(\Delta)$ be the family of all analytic functions in the open unit disc $\Delta:=$ $\{z:|z|<1\}$. Let $f$ and $g$ be two members of the class $\mathcal{H}(\Delta)$ which satisfy the normalized conditions $f(0)=0=f^{\prime}(0)-1$ and $g(0)=0=g^{\prime}(0)-1$. We say that a function $f$ is subordinate to $g$, written $f(z) \prec g(z)$ or $f \prec g$, if there exists an analytic function $\psi$, known as a Schwarz function, with $\psi(0)=0$ and $|\psi(z)| \leq|z|$ such that $f(z)=g(\psi(z))$ for all $z \in \Delta$. The set of all univalent (one-to-one) functions $f$ in $\Delta$ is denoted by $\mathcal{U}$. Furthermore, if $g \in \mathcal{U}$ in $\Delta$, then we have the following equivalence relation

$$
f(z) \prec g(z) \Leftrightarrow f(0)=g(0) \text { and } \quad f(\Delta) \subset g(\Delta) .
$$

Also let $\mathcal{B}(\Delta)$ denote the set of all functions $w \in \mathcal{H}(\Delta)$ satisfying $|w(z)|<1$ in $\Delta$. A mapping $f$ is said to be $\log -$ harmonic in $\Delta$, if there exists an analytic function $w \in \mathcal{B}(\Delta)$ such that $f$ is a solution of the nonlinear elliptic partial differential equation

$$
\frac{\overline{f_{\bar{z}}}}{\bar{f}}=w(z) \frac{f_{z}}{f}
$$

where $w$ is the second complex dilatation of $f$ and $w \in \mathcal{B}(\Delta)$. We note that if $f$ is a non-vanishing log-harmonic mapping, then $f$ has the following form

$$
f(z)=h(z) \overline{g(z)},
$$

where both $h$ and $g$ are non-vanishing analytic functions, and that if $f$ vanishes at $z=0$ but is not identically zero, then $f$ has the following form

$$
f(z)=z|z|^{2 \beta} h(z) \overline{g(z)},
$$

2010 Mathematics Subject Classification. 30C35;30C45;35Q30.

Key words and phrases. starlike log-harmonic mappings; log-harmonic mappings; subordination; Jacobian; real part. 
where $\operatorname{Re}\{\beta\}>-1 / 2, h$ and $g$ are in $\mathcal{H}(\Delta), h(0) \neq 0$ and $g(0)=1$ (see [3]). The class of functions of this form has been studied extensively by many works, see for example [1]- 6] and 9].

We continue the discussion with an example.

Example 1.1. If $f(1)=1$ and $\operatorname{Re}\{\beta\}>-1 / 2$, then the function

$$
f_{\beta}(z)=z|z|^{2 \beta} \quad(z \in \Delta),
$$

is a solution of the equation (1.1) in the complex plane $\mathbb{C}$ with $w \equiv \bar{\beta} /(1+\beta)$. It is a simple exercise that $f_{\beta}$ maps the unit disc $\Delta$ onto itself. The Figure 1 shows the image of the unit disc $\Delta$ under the function $f_{\beta}$ in two different cases.

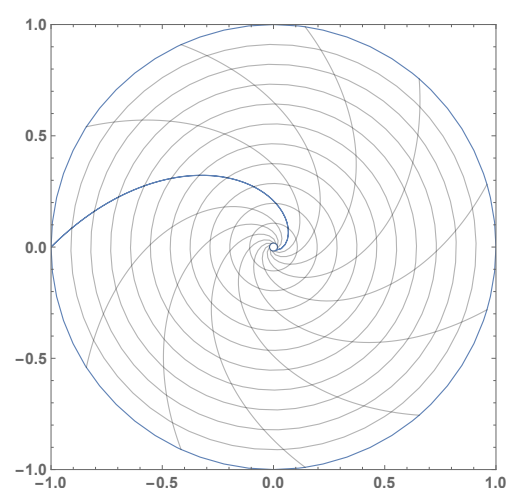

(a)

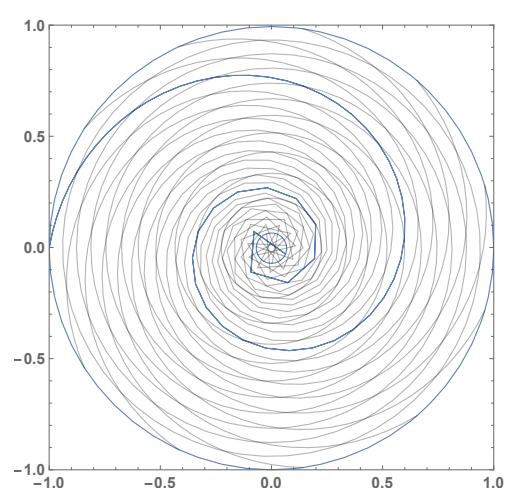

(b)

FiguRE 1. (a) The boundary curve of $f_{i}(\Delta)(\mathrm{b})$. The boundary curve of $f_{-1 / 3+4 i}(\Delta)$

We denote by $J_{f}(z)$ the Jacobian of log-harmonic mappings $f$ as follows

$$
J_{f}(z)=\left|f_{z}\right|^{2}\left(1-|w(z)|^{2}\right) .
$$

Since $w \in \mathcal{B}(\Delta)$, thus $J_{f}(z)>0$ and all non-constant log-harmonic mappings are sense-preserving and open in the disc $\Delta$.

Let $f(z)=z|z|^{2 \beta} h(z) \overline{g(z)}$, where $h(0)=g(0)=1$, be a log-harmonic mapping. Then we say that $f$ is a starlike log-harmonic $(\mathcal{S L H})$ mapping of order $\alpha$, where $0 \leq \alpha<1$, if

$$
\frac{\partial}{\partial \theta} \arg f\left(r e^{i \theta}\right)=\operatorname{Re}\left\{\frac{z f_{z}-\bar{z} f_{\bar{z}}}{f}\right\}>\alpha \quad(z \in \Delta) .
$$

We denote by $\mathcal{S}_{\mathcal{L} \mathcal{H}}^{*}(\alpha)$, the set of all starlike log-harmonic mappings of order $\alpha$.

The following lemma will be useful.

Lemma 1.1. 8, p. 35] Let $\Xi$ be a simply connected domain in the complex plane $\mathbb{C}, \Xi \neq \mathbb{C}$ and let $b$ be a complex number with $\operatorname{Re}\{b\}>0$. Suppose that a function $\psi: \mathbb{C}^{2} \times \Delta \rightarrow \mathbb{C}$ satisfies the condition

$$
\psi(i \rho, \sigma ; z) \notin \Xi
$$

for all real $\rho, \sigma \leq-|b-i \rho|^{2} /(2 \operatorname{Re}\{b\})$ and all $z \in \Delta$. If the function $p(z)$ defined by $p(z)=b+b_{1} z+b_{2} z^{2}+\cdots$ is analytic in $\Delta$ and if

$$
\psi\left(p(z), z p^{\prime}(z) ; z\right) \in \Xi \text {, }
$$

then $\operatorname{Re}\{p(z)\}>0$ in $\Delta$. 
One of the goals of this paper is to investigate some geometric properties of the starlike $\log$-harmonic mappings of order $\alpha$, another is to give an estimate for the Jacobian of $\log$-harmonic mappings of the form 1.2 .

\section{Main Results}

In the first result, by use of the subordination principle, we give a necessary and sufficient condition for functions of the form 1.2 belonging to the class $\mathcal{S}_{\mathcal{L} \mathcal{H}}^{*}(\alpha)$.

Theorem 2.1. Let $\alpha \in[0,1)$ and $\operatorname{Re}\{\beta\}>-1 / 2$. Then the function $f(z)=$ $z|z|^{2 \beta} h(z) \overline{g(z)}$ belongs to the class $\mathcal{S}_{\mathcal{L H}}^{*}(\alpha)$ if, and only if,

$$
\left(z \frac{h^{\prime}(z)}{h(z)}-z \frac{g^{\prime}(z)}{g(z)}\right) \prec \frac{2(1-\alpha) z}{1-z} \quad(z \in \Delta) .
$$

Proof. Let the function $f(z)=z|z|^{2 \beta} h(z) \overline{g(z)}$ belongs to the class $\mathcal{S}_{\mathcal{L} \mathcal{H}}^{*}(\alpha)$ where $0 \leq \alpha<1$. Then by a simple check we get

$$
\frac{z f_{z}}{f}=1+\beta+z \frac{h^{\prime}(z)}{h(z)} \quad(z \in \Delta)
$$

and

$$
\frac{\bar{z} f_{\bar{z}}}{f}=\beta+\bar{z}\left(\frac{g^{\prime}(z)}{g(z)}\right) \quad(z \in \Delta)
$$

Thus

$$
\frac{z f_{z}-\bar{z} f_{\bar{z}}}{f}=1+z \frac{h^{\prime}(z)}{h(z)}-\overline{z\left(\frac{g^{\prime}(z)}{g(z)}\right)} \quad(z \in \Delta) .
$$

Then $f \in \mathcal{S}_{\mathcal{L H}}^{*}(\alpha)$ if, and only if,

$$
\begin{aligned}
\alpha<\operatorname{Re}\left\{\frac{z f_{z}-\bar{z} f_{\bar{z}}}{f}\right\} & =\operatorname{Re}\left\{1+z \frac{h^{\prime}(z)}{h(z)}-\bar{z} \overline{\left(\frac{g^{\prime}(z)}{g(z)}\right)}\right\} \\
& =\operatorname{Re}\left\{1+z \frac{h^{\prime}(z)}{h(z)}-z \frac{g^{\prime}(z)}{g(z)}\right\} .
\end{aligned}
$$

Therefore

$$
\operatorname{Re}\left\{z \frac{h^{\prime}(z)}{h(z)}-z \frac{g^{\prime}(z)}{g(z)}\right\}>\alpha-1 \quad(z \in \Delta) .
$$

Now by use of the subordination principle we get

$$
\operatorname{Re}\left\{z \frac{h^{\prime}(z)}{h(z)}-z \frac{g^{\prime}(z)}{g(z)}\right\} \prec \frac{2(1-\alpha) z}{1-z} \quad(z \in \Delta) .
$$

This ends the proof.

We now have the following lemma directly.

Lemma 2.1. The function $f(z)=z|z|^{2 \beta} h(z) \overline{g(z)}(\operatorname{Re}\{\beta\}>-1 / 2)$ belongs to the class $\mathcal{S}_{\mathcal{L H}}^{*}(\alpha)$ if, and only if,

$$
1+\operatorname{Re}\left\{z \frac{h^{\prime}(z)}{h(z)}-z \frac{g^{\prime}(z)}{g(z)}\right\}>\alpha \quad(0 \leq \alpha<1, z \in \Delta) .
$$

Following, we give the representation theorem for the function $h$ of the mappings $f$ of the form 1.2 in the set $\mathcal{S}_{\mathcal{L} \mathcal{H}}^{*}(\alpha)$. 
Theorem 2.2. A function $f(z)=z|z|^{2 \beta} h(z) \overline{g(z)}$ belongs to the class $\mathcal{S}_{\mathcal{L} \mathcal{H}}^{*}(\alpha)$ if, and only if,

$$
h(z)=g(z) \exp \left(\int_{0}^{z} \frac{2(1-\alpha) \psi(t)}{t(1-\psi(t))} \mathrm{d} t\right) \quad(z \in \Delta),
$$

where $\psi$ is a Schwarz function, $0 \leq \alpha<1$ and $\operatorname{Re}\{\beta\}>-1 / 2$.

Proof. If $f(z)=z|z|^{2 \beta} h(z) \overline{g(z)}$ belongs to the class $\mathcal{S}_{\mathcal{L} \mathcal{H}}^{*}(\alpha)$ where $0 \leq \alpha<1$, then by Theorem 2.1 and by definition of subordination, there exists a Schwarz function $\psi$ such that

or equivalently

$$
\left(z \frac{h^{\prime}(z)}{h(z)}-z \frac{g^{\prime}(z)}{g(z)}\right)=\frac{2(1-\alpha) \psi(z)}{1-\psi(z)} \quad(z \in \Delta),
$$

$$
\left\{\log \frac{h(z)}{g(z)}\right\}^{\prime}=\frac{2(1-\alpha) \psi(z)}{z(1-\psi(z))} \quad(z \in \Delta) .
$$

Integrating the last equality 2.7), we get 2.6). On the other hand, it is an easy calculation that a function having of the form (2.6) satisfies the condition (2.1).

Applying formula 2.6 for $\psi(z)=z$ gives the following.

Example 2.1. Let $g(z)$ be an analytic function with $g(0)=1$. Then the function

$$
F_{\alpha, \beta}(z)=z|z|^{2 \beta} \frac{|g(z)|^{2}}{(1-z)^{2(1-\alpha)}} \quad(z \in \Delta, 0 \leq \alpha<1),
$$

is a starlike log-harmonic mapping of order $\alpha$.

Remark 2.1. Since $g(0)=1$, thus if we consider the constant function $g(z)=1$ in (2.8) and let $\beta=0$, then we obtain

$$
f(z)=\frac{z}{(1-z)^{2(1-\alpha)}} \quad(0 \leq \alpha<1) .
$$

Moreover, the Koebe function of order $\alpha$ (in particular, the Koebe function) belongs to the class $\mathcal{S}_{\mathcal{L} \mathcal{H}}^{*}(\alpha)$.

Example 2.2. Let $\psi(z)=z /(1+z)$ and $|z|<0.65$. Then it is easy to see that $\psi$ is Schwarz function. By putting $\psi(z)=z /(1+z)$ in the equation (2.6), we get

$$
h(z)=g(z) e^{2(1-\alpha) z} \quad(|z|<0.65) .
$$

Thus the function

$$
f_{\alpha, \beta}(z)=z|z|^{2 \beta} e^{2(1-\alpha) z}|g(z)|^{2} \quad(\operatorname{Re}\{\beta\}>-1 / 2,|z|<0.65),
$$

belongs to the class $S T_{L H}(\alpha)$, where $g$ is analytic with $g(0)=1$. In particular, the function

$$
\widehat{f}_{\alpha, \beta}(z)=z|z|^{2 \beta} e^{2(1-\alpha) z} \quad(|z|<0.65),
$$

is a starlike log-harmonic mapping of order $\alpha$ where $\operatorname{Re}\{\beta\}>-1 / 2$. Also, $\widehat{f}_{0,0}(z)=$ $z e^{2 z}(|z|<0.65)$ is $\mathcal{S} \mathcal{L H}$ mapping. It is a simple exercise that the radius of injectivity of the function $\widehat{f}_{0,0}$ is $1 / 2$. The Figures $2(a)$ and $2(b)$ show the image of the disc $|z|<0.65$ and $|z|<1 / 2$ under the function $\widehat{f}_{0,0}$, respectively.

Theorem 2.3. Let $\alpha \in[0,1)$ and $\operatorname{Re}\{\beta\}>-1 / 2$. Let a function $f(z)=z|z|^{2 \beta} h(z) \overline{g(z)}$ belongs to the class $\mathcal{S}_{\mathcal{L} \mathcal{H}}^{*}(\alpha)$ where

$$
h(z)=1+\sum_{n=1}^{\infty} a_{n} z^{n} \text { and } g(z)=1+\sum_{n=1}^{\infty} b_{n} z^{n},
$$




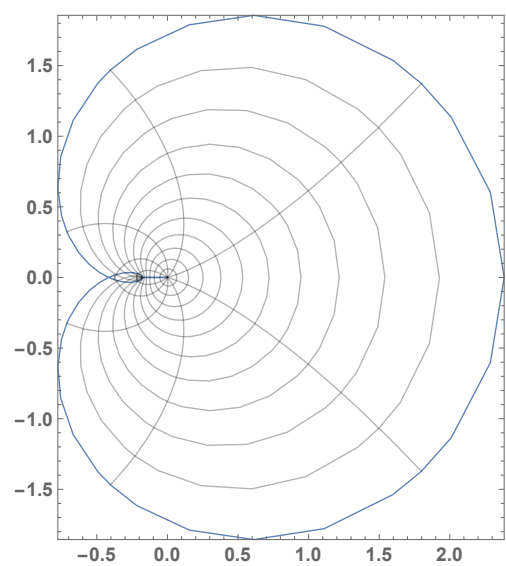

(a)

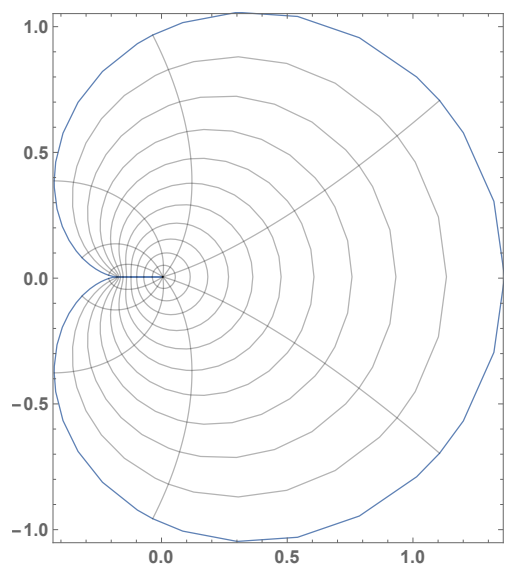

(b)

Figure 2. (a) The boundary curve of $\widehat{f}_{0,0}(|z|<0.65)$ (b) The boundary curve of $\widehat{f}_{0,0}(|z|<1 / 2)$

be a log-harmonic mapping, and let

$$
\frac{z h^{\prime}(z)}{h(z)}=\sum_{n=1}^{\infty} h_{n} z^{n} \quad \text { and } \quad \frac{z g^{\prime}(z)}{g(z)}=\sum_{n=1}^{\infty} g_{n} z^{n} .
$$

If

$$
\sum_{n=1}^{\infty}\left|h_{n}\right|<1-|\beta|
$$

and

$$
\sum_{n=1}^{\infty}\left(\left|h_{n}\right|+\left|g_{n}\right|\right) \leq 1-2|\beta|
$$

then $f$ is sense preserving.

Proof. To show that $f$ is sense preserving, we need to prove that $|w(z)|<1$ where $w$ denotes the dilatation of $f$. A simple calculation using 1.1$)$ and $(1.2)$, gives us

$$
w(z)=\frac{\overline{f_{\bar{z}}}}{\bar{f}} \frac{f}{f_{z}}=\frac{\bar{\beta}+z \frac{g^{\prime}(z)}{g(z)}}{1+\beta+z \frac{h^{\prime}(z)}{h(z)}} \quad(z \in \Delta) .
$$

Clearly $w(0)=\bar{\beta} /(1+\beta)=: \gamma$, where $\beta \in \mathbb{C}$ and $\operatorname{Re}\{\beta\}>-1 / 2$. We have

$$
\begin{aligned}
|w(z)| & =\left|\frac{\bar{\beta}+z \frac{g^{\prime}(z)}{g(z)}}{1+\beta+z \frac{h^{\prime}(z)}{h(z)}}\right| \\
& =\left|\frac{\bar{\beta}+\sum_{n=1}^{\infty} g_{n} z^{n}}{1+\beta+\sum_{n=1}^{\infty} h_{n} z^{n}}\right| \\
& \leq \frac{|\bar{\beta}|+\sum_{n=1}^{\infty}\left|g_{n}\right||z|^{n}}{1-|\beta|-\sum_{n=1}^{\infty}\left|h_{n}\right||z|^{n}} \\
& <\frac{|\bar{\beta}|+\sum_{n=1}^{\infty}\left|g_{n}\right|}{1-|\beta|-\sum_{n=1}^{\infty}\left|h_{n}\right|} \leq 1 .
\end{aligned}
$$

This proves the theorem. 
To prove the following theorem, we will use the method of [7, Theorem 2.1].

Theorem 2.4. Let $\alpha \in(1 / 2,1)$. If the function $f(z)=z|z|^{2 \beta} h(z) \overline{g(z)}$ belongs to the class $\mathcal{S}_{\mathcal{L} \mathcal{H}}^{*}(\alpha)$, then

$$
\operatorname{Re}\left\{\frac{h(z)}{g(z)}\right\}>\mu(\alpha):=\frac{1}{3-2 \alpha} \quad(z \in \Delta) .
$$

Proof. For convenience, we put $\mu(\alpha):=\mu$ and note that $\mu \in(1 / 2,1)$ when $\alpha \in$ $(1 / 2,1)$. Define

$$
p(z)=\frac{1}{1-\mu}\left(\frac{h(z)}{g(z)}-\mu\right) \quad(z \in \Delta) .
$$

Then $p(z)$ is analytic function in $\Delta$ and $p(0)=1$. A simple check gives

$$
1+z \frac{h^{\prime}(z)}{h(z)}-z \frac{g^{\prime}(z)}{g(z)}=1+\frac{(1-\mu) z p^{\prime}(z)}{\mu+(1-\mu) p(z)}=: \phi\left(p(z), z p^{\prime}(z)\right)
$$

where

$$
\phi(x, y)=1+\frac{(1-\mu) y}{\mu+(1-\mu) x} .
$$

By Lemma 2.1, since $f(z)=z|z|^{2 \beta} h(z) \overline{g(z)} \in \mathcal{S}_{\mathcal{L H}}^{*}(\alpha)$, we can consider

$$
\left\{\phi\left(p(z), z p^{\prime}(z)\right): z \in \Delta\right\} \subset\{w \in \mathbb{C}: \operatorname{Re}\{w\}>\alpha\}=: \Omega_{\alpha} .
$$

Now for all real $\rho$ and $\sigma \leq-\frac{1}{2}\left(1+\rho^{2}\right)$, we get

$$
\begin{aligned}
\operatorname{Re}\{\phi(i \rho, \sigma)\} & =\operatorname{Re}\left\{1+\frac{(1-\mu) \sigma}{\mu+(1-\mu) i \rho}\right\} \\
& =1+\frac{\mu(1-\mu) \sigma}{\mu^{2}+(1-\mu)^{2} \rho^{2}} \\
& \leq 1-\frac{1}{2} \mu(1-\mu) Q(\rho),
\end{aligned}
$$

where

$$
Q(\rho):=\frac{1+\rho^{2}}{\mu^{2}+(1-\mu)^{2} \rho^{2}} .
$$

It is easy to see that

$$
Q^{\prime}(\rho)=\frac{2(2 \mu-1) \rho}{\left(\mu^{2}+(1-\mu)^{2} \rho^{2}\right)^{2}}
$$

and $Q^{\prime}(0)=0$ occurs at only $\rho=0$ and satisfies $Q(0)=1 / \mu^{2}$. Also

$$
\lim _{\rho \rightarrow \pm \infty} Q(\rho)=\frac{1}{(1-\mu)^{2}} \text {. }
$$

Thus we have

$$
\frac{1}{\mu^{2}} \leq Q(\rho)<\frac{1}{(1-\mu)^{2}} \quad(1 / 2<\mu<1)
$$

Hence

$$
\operatorname{Re}\{\phi(i \rho, \sigma)\} \leq 1-\frac{1}{2} \mu(1-\mu) \frac{1}{\mu^{2}}=\frac{3 \mu-1}{2 \mu}=\alpha
$$

and this shows that $\operatorname{Re}\{\phi(i \rho, \sigma)\} \notin \Omega_{\alpha}$. Moreover, by Lemma 1.1 we get $\operatorname{Re}\{p(z)\}>$ 0 in $\Delta$, and concluding the proof.

Finally, we give an estimate for the Jacobian of log-harmonic mappings of the form 1.2 . 
Theorem 2.5. If $f(z)=z|z|^{2 \beta} h(z) \overline{g(z)}$ is log-harmonic mapping, then

$$
\frac{\left(1-|\gamma|^{2}\right)\left(1-|z|^{2}\right)}{(1+|\gamma||z|)^{2}}\left|f_{z}\right|^{2} \leq J_{f}(z) \leq \begin{cases}\frac{\left(1-|\gamma|^{2}\right)\left(1-|z|^{2}\right)}{(1-|\gamma||z|)^{2}}\left|f_{z}\right|^{2} & |z|<|\gamma|, \\ \left|f_{z}\right|^{2} & |z| \geq|\gamma|,\end{cases}
$$

where $\gamma=\bar{\beta} /(1+\beta), \beta \in \mathbb{C}, \operatorname{Re}\{\beta\}>-1 / 2$ and $z \in \Delta$.

Proof. Let $\gamma=\bar{\beta} /(1+\beta), \beta \in \mathbb{C}$ and $\operatorname{Re}\{\beta\}>-1 / 2$. Consider the function

$$
\varphi(z):=\frac{w(z)-\gamma}{1-\bar{\gamma} w(z)} \quad(z \in \Delta)
$$

where $w$ is the dilatation of $f$ of the form 1.1 . We have that $\varphi$ is analytic (because $|1+\bar{\beta}|>|\beta|), \varphi(0)=0$ and $|\varphi(z)| \leq 1$. Thus the function $\varphi$ satisfies the assumptions of Schwarz lemma which gives $|\varphi(z)| \leq|z|$ or

$$
|w(z)-\gamma| \leq|z||1-\bar{\gamma} w(z)| \quad(z \in \Delta) .
$$

From 2.16), we get

$$
w(z)=\frac{\varphi(z)+\gamma}{1+\bar{\gamma} \varphi(z)} .
$$

This shows that the dilatation $w(z)$ is subordinate to

$$
\phi(z)=\frac{z(z+\gamma)}{1+\bar{\gamma} z}
$$

Since the linear transformation $\phi(z)$ maps $|z|=r$ onto the disc with the center

$$
\left(\frac{x\left(1-|z|^{2}\right)}{1-|\gamma|^{2}|z|^{2}}, \frac{y\left(1-|z|^{2}\right)}{1-|\gamma|^{2}|z|^{2}}\right) \quad(x=\operatorname{Re}\{\gamma\}, y=\operatorname{Im}\{\gamma\})
$$

and the radius

$$
\frac{\left(1-|\gamma|^{2}\right)|z|}{1-|\gamma|^{2}|z|^{2}}
$$

therefore, by use of the above and by the subordination principle, the inequality 2.17) gives that

and concluding the proof.

$$
\left|w(z)-\frac{\gamma\left(1-|z|^{2}\right)}{1-|\gamma|^{2}|z|^{2}}\right| \leq \frac{\left(1-|\gamma|^{2}\right)|z|}{1-|\gamma|^{2}|z|^{2}}
$$

Acknowledgements. The authors would like to thank the anonymous referee(s) for carefully reading of the manuscript and for the useful observations.

\section{REFERENCES}

[1] Z. Abdulhadi, Close-to-starlike log-harmonic mappings, Internat. J. Math. Math. Sci. 19(3), 563-574 (1996)

[2] Z. Abdulhadi, Typically real log-harmonic mappings, Internat. J. Math. Math. Sci. 31(1), 1-9 (2002)

[3] Z. Abdulhadi and D. Bshouty, Univalent functions in $H \bar{H}$, Tran. Amer. Math. Soc. 305(2), 841-849 (1988)

[4] Z. Abdulhadi and W. Hergartner, Spirallike logharmonic mappings, Complex Variables Theory Appl. 9(2-3), 121-130 (1987)

[5] Z. Abdulhadi and W. Hergartner, One pointed univalent logharmonic mappings, J. Math. Anal. Appl. 203(2), 333-351 (1996)

[6] R. M. Ali, Z. Abdulhadi and Ch. Zhen, The Bohr radius for starlike logharmonic mappings, Complex Var. Elliptic Equ. 61(1), 1-14 (2016)

[7] R. Kargar, A. Ebadian and J. Sokól, On subordination of some analytic functions, Sib. Math. J. 57(4), 599-604 (2016)

[8] S.S. Miller and P.T. Mocanu, Differential Subordinations, Theory and Applications, Series of Monographs and Textbooks in Pure and Applied Mathematics, Vol. 225, Marcel Dekker Inc., New York / Basel 2000. 
[9] Zh. Mao, S. Ponnusamy, and X. Wang, Schwarzian derivative and Landau's theorem for logharmonic mappings, Complex Var. Elliptic Equ. 58(8), 1093-1107 (2013)

Young Researchers and Elite Club, Ardabil Branch, Islamic Azad University, ArdABIL, IRAN

E-mail address: rkargar1983@gmail.com

Department of Mathematics, Firoozkouh Branch, Islamic Azad University, FiroozkOUH, IRAN

E-mail address: hesammahzoon1@gmail.com 